\title{
BIOESTRATIGRAFIA DE LA FORMACION CANSONA EN LA QUEBRADA PEÑITAS, CINTURON DE SAN JACINTO. IMPLICACIONES PALEOGEOGRÁFICAS
}

\author{
BIOSTRATIGRAPHY OF THE CANSONA FORMATION AT \\ QUEBRADA PEÑITAS, SAN JACINTO BELT. PALEOGEOGRAPHIC \\ IMPLICATIONS
}

\author{
Hernando Dueñas Jiménez PhD.*, Clemencia Gómez González PhD.**
}

RESUMEN

Dueñas Jiménez H., C. Gómez González: Bioestratigrafía de la Formación Cansona en la quebrada Peñitas, cinturon de San Jacinto. Implicaciones paleogeográficas. Rev. Acad. Colomb. Cienc. 37 (145): 527-539, 2013. ISSN 0370-3908.

En este trabajo se presentan los resultados de los análisis bioestratigráficos de trece muestras de la Formación Cansona recolectadas a lo largo del Quebrada Peñitas, la cual corta el núcleo del Anticlinal de Chalán que queda localizado en el Cinturón de San Jacinto, región Caribe Colombiana.

Con base en la información obtenida, se les asigna a los sedimentos de la Formación Cansona una edad Cretáceo Tardío. A la parte basal de la sección analizada se le asigna una edad Campaniano Tardío a Maastrichtiano Temprano. A la parte superior, se le asigna una edad Maastrichtiano Tardío. La totalidad de los sedimentos analizados fueron depositados en ambientes marinos cercanos a la costa, los cuales recibieron gran aporte de terrígenos. Se observa una somerización de la cuenca de depósito a medida que se asciende estratigráficamente.

La microfauna recolectada solo incluye foraminíferos bentónicos de amplio rango estratigráfico dentro del Cretáceo Tardío, los cuales son indicadores de ambientes marinos, pero poco aportan a la definición de edades.

De las muestras analizadas de la parte superior de la Formación Cansona, fue posible recuperar buenas asociaciones de Polen y de esporas, las cuales incluyen Buttinia andreevi, Zlivisporites blanensis y Proxapertites humbertoides entre muchos otros. Esta asociacion de palinomorfos nos permite determinar que la parte superior de la secuencia estratigráfica analizada pertenece a la Zona de Proteacidites dehaani (Germeraad et al., 1968) de edad Maastrichtiano Tardío.

Muy buenas asociaciones de dinoflagelados fueron recuperadas de las muestras analizadas de la parte inferior de esta secuencia. Estas asociaciones de palinomorfos se caracterizan por la co-ocurrencia de especies de los

* $\quad$ BIOSS SAS hdjbioss@yahoo.com

** Agencia Nacional de Hidrocarburos(A.N.H.). Ggc06@Anh.gov.co 
géneros Andalusiella, Cerodinium y Senegalinium lo cual es indicativo de que estos sedimentos pertenecen al Paleocinturón Tropical (Malloy Suite) establecido por Lentin y Williams (1980).

Asociaciones palinológicas similares a las obtenidas de muestras de la Formación Cansona, en la Quebrada Peñitas, han sido reportadas a lo largo y ancho de la cordillera oriental (Fig. 4).

En la región del Cinturón de San Jacinto durante el Cretáceo Tardío, se presentó un mar tropical somero (mar Cansona) que se encontraba interconectado con los mares que permitieron el depósito de los sedimentos de las Formaciones Colón, Umir y Formaciones equivalentes.

Por primera vez se reporta la presencia de sedimentos pertenecientes a la zona de Proteacidites dehaani y del denominado Paleocinturón Tropical al occidente de la Sutura de Romeral.

Palabras clave: dinoflagelados Cretaceo Superior, Formación Cansona, Foraminiferos, Paleo-ambiente, Paleocinturon Tropical.

\section{SUMMARY}

In this paper the results obtained from the biostratigraphical analysis of 13 samples from the Cansona Formation are presented. These samples were collected along the Quebrada Peñitas. The Quebrada Peñitas cuts through the core of the Chalán anticline and it is located on the San Jacinto belt of the Caribbean region of Colombia.

Based on the information obtained, it is possible to assign a Late Cretaceous age to the Casona Formation. For the lower part of the analyzed section, it is possible to assign a Late Campanian to Early Maastrichtian age. For the upper part, we assigned a Late Maastrichtian age. According to the micropaleontological content, all of the analyzed sediments were deposited on shallow marine near-coast environments, which received a great influx of terrigenous material. It is possible to observe a shallowing of the basin as we ascend stratigraphically.

The microfauna collected includes only long-range Cretaceous benthonic foraminifera, which are indicative of marine environments but do not contribute much in assigning a more precise age.

From the analysed samples of the upper part of the section, it was possible to obtain good assemblages of pollen and spores, including Buttinia andreevi, Zivisporites blanensis and Proxapertites humbertoides, among many others. These assemblages allow us to assign to this part of the section a Late Maastrichtian age, and assume that these sediments belong to the Proteacidites dehaani zone (Germeraad et al., 1968).

Very good assemblages of dinoflagellates were recovered from samples collected on the lower part of the analysed section. They are characterized by the co-occurrence of species of the genera Andalusiella, Cerodinium and Senegalinium, which are indicative that these sediments belong to the Tropical Paleo-Belt (Malloy suite), as established by Lentin and Williams (1980).

The palynological assemblages similar to those recovered from sediments belonging to Quebrada Peñitas have been widely reported throughout the Eastern Cordillera. During the late Cretaceous Period there was a shallow Tropical sea (Cansona Sea), in the San Jacinto belt region, that was interconnected with the seas that allowed the deposition of the sediments belonging to the Colon, Umir, and equivalent Units.

In this paper is reported, for the first time, the presence of sediments belonging to the Proteacidites dehaani and to the Tropical Paleo-Belt located west of the current Romeral Fault.

Key words: upper Cretaceous Dinoflagellates, Cansona Formation, Foraminifera, Paleoenvironment, Tropical Paleo-Belt.

\section{Introducción}

El Cinturón plegado de San Jacinto, es una faja orogénica que corre paralela a la costa del Caribe en la parte Nor-Occidental de Colombia. Limita al Occidente con el cinturón plegado del Sinú (Cuenca del Sinú). Al Oriente lo bordea la Falla (Sutura) de Romeral que, al mismo tiempo, le sirve de límite con la Cuenca del Valle Inferior del Magdalena (Fig.1).

El término estratigráfico Formación Cansona (Notestein \& Aitken (1929); en Duque Caro et al., 1996) hace relación 


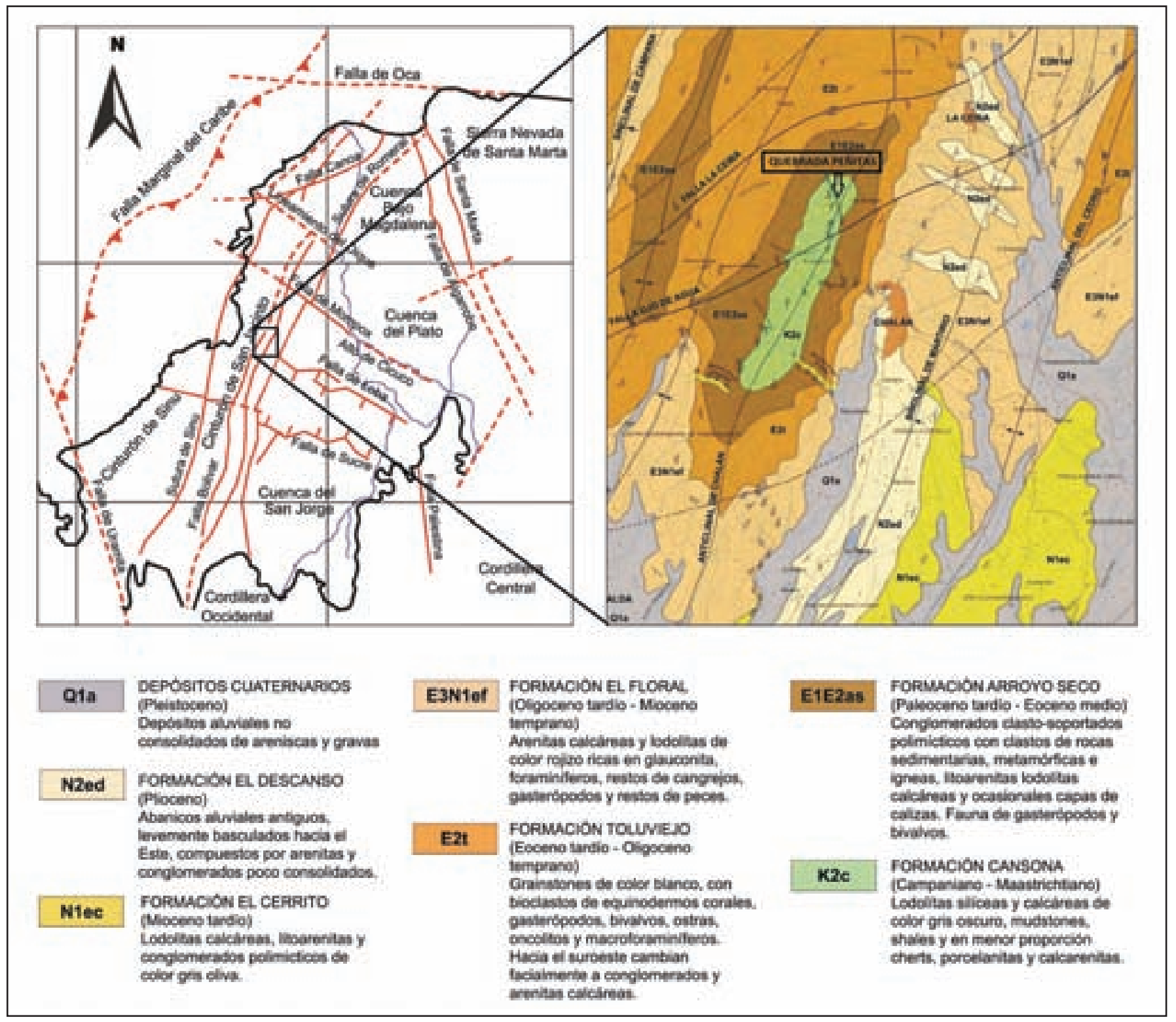

Figura 1. Localización regional y mapa geológico del anticlinal de Chalán

a una secuencia de $230 \mathrm{~m}$ de espesor que aflora en el Cerro Cansona (Departamento de Bolívar), localizado a $35 \mathrm{~km}$ al Noroccidente de la población de Chalán. En el área de estudio, la Formación Cansona aflora formando el núcleo del anticlinal de Chalán (Fig.1). Con base en la microfauna recuperada de muestras provenientes de varias secciones en el Cerro Cansona, la Texas Petroleum Company le asignó a la Formación Cansona en 1942 (Duque Caro, 1972) una edad de Santoniano a Maastrichtiano, y un ambiente de depósito marino somero. Hacia la parte inferior de la formación, la Texas Petroleum reportó la co-ocurrencia de Heterohelix globulosa, Globotruncana spp., Bulimina spp., y Globige- rinoides spp. Hacia el tope reportó la común presencia de Siphogenerinoides cretacea. En los estudios llevados a cabo, la microfauna recobrada de las muestras de la Formación Cansona en la Quebrada Peñitas se compone exclusivamente de foraminiferos bentónicos, lo cual nos ha impedido corroborar la edad asignada por la Texas Petroleum Company.

En todos los sitios de afloramiento, las rocas de la Formación Cansona se presentan como pequeños remanentes sedimentarios aislados tectónicamente. Dueñas \& Duque (1981) mencionan que los sedimentos de la Formación Cansona descansan discordantemente sobre basamento de tipo oceá- 
nico (i.e., sobre las Peridotitas de Planeta Rica). Por su parte, Clavijo \& Barrera (2001) mencionan que estos sedimentos se encuentran en contacto directo con unas diabasas de textura amigdaloide (Fig. 2). Posterior al depósito de las rocas de la Formación Cansona, se presenta el inicio de la Orogenia Pre-Andina (Duque Caro, 1972), razón por la cual el contacto superior con la suprayacente Formación Arroyo Seco es claramente discordante (Fig. 2).

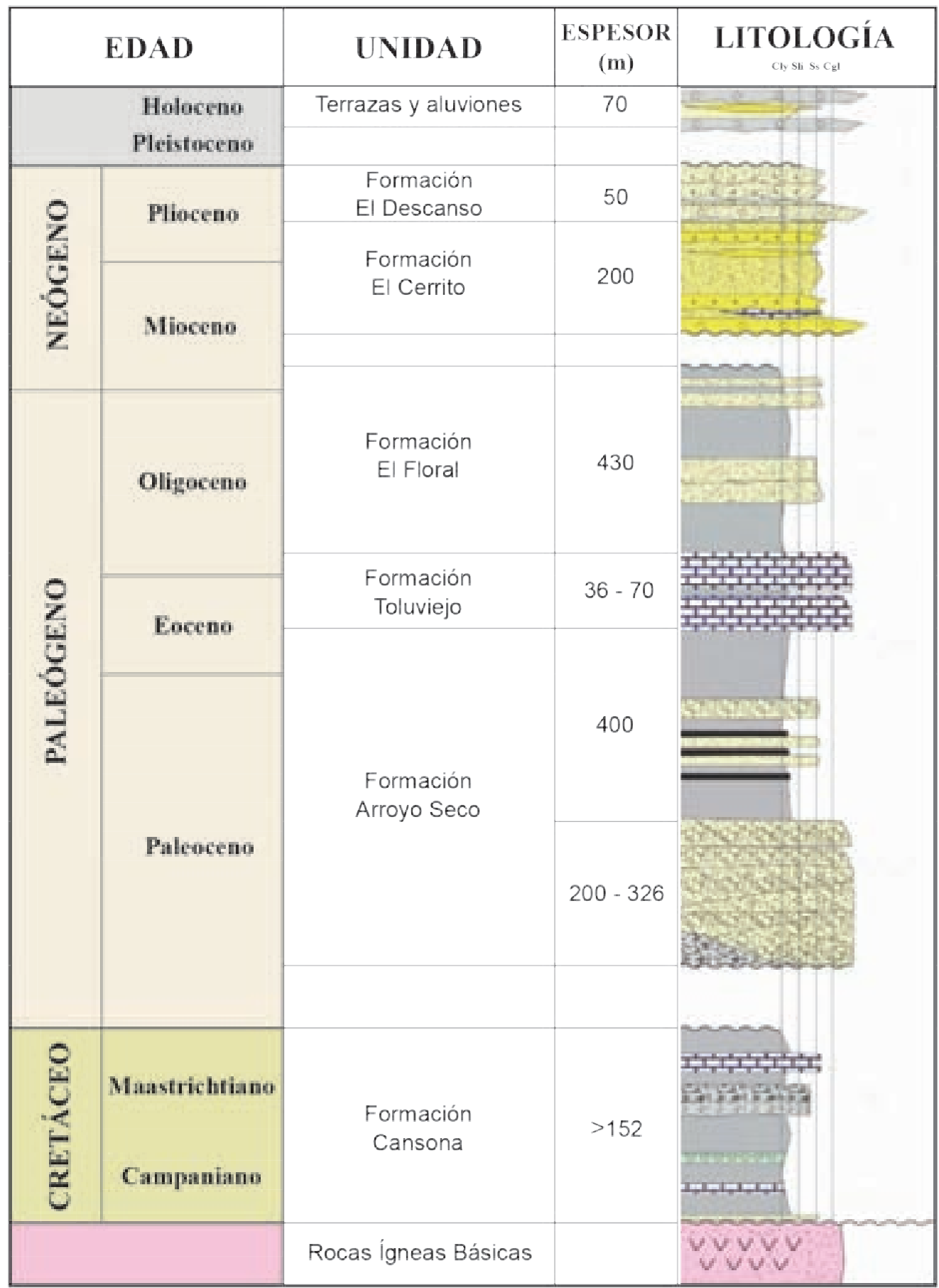

Figura 2. Columna estratigráfica generalizada pra el cinturón de San Jacinto. 
Muestras de la Formación Cansona provenientes de varias secciones han sido analizadas por métodos geoquímicos, reportando valores de carbono orgánico total que varían entre el 2 y 12\% (con promedios de 4\%). El querógeno predominante es tipo I y II (i.e., de origen marino). Los valores de reflectancia de vitrinita alcanzan $0.77 \%$, y las temperaturas máximas de pirolisis están alrededor de $440^{\circ} \mathrm{C}$. Estos valores geoquímicos permiten considerar a los sedimentos de la Formación Cansona como las rocas generadoras de hidrocarburos del Sistema Petrolero del Cinturón plegado de San Jacinto (Bioss, 2005).

\section{Materiales y métodos utilizados}

La Formación Cansona aflora en el núcleo del anticlinal de Chalán. En la parte más norte del Anticlinal de Chalán, la Quebrada Peñitas atraviesa una sección de 152 metros perteneciente a esta formación (Fig.1). Por su parte, la Figura 3 presenta la columna estratigráfica levantada a lo largo de la Quebrada Peñitas. En ella se localizan las trece muestras que fueron preparadas y analizadas por métodos palinológicos y micropaleontológicos. Adicionalmente en esta gráfica, se presentan las edades establecidas para estos sedimentos y la subdivisión palinológica propuesta.

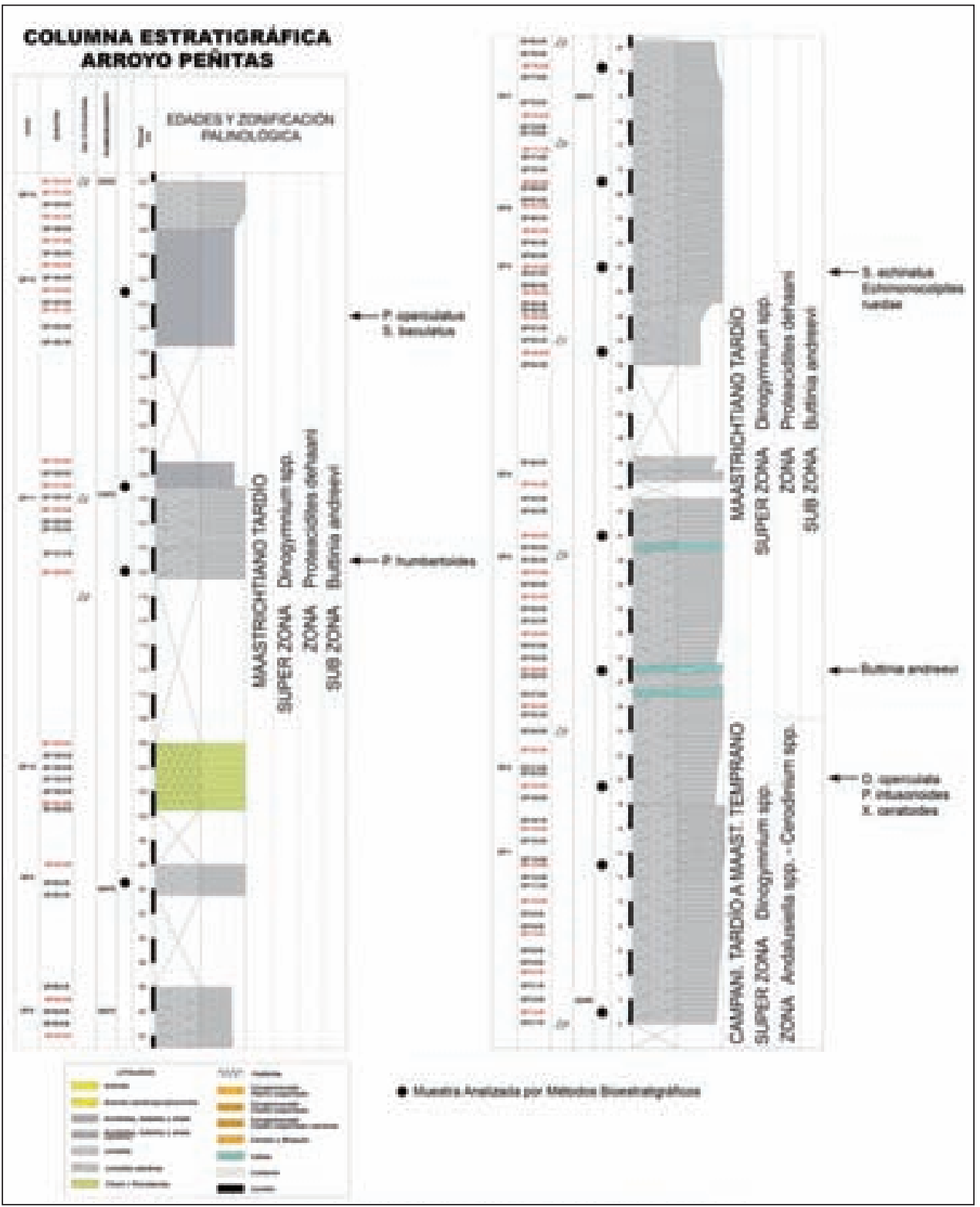

Figura 3. Columna estratigráfica de la Formación Cansona en la quebrada Peñitas. 
Las muestras seleccionadas para determinaciones palinológicas fueron preparadas utilizando el método de disgregación, el cual consiste en separar (apartar) los componentes sedimentológicos de las rocas, eliminando el cemento que los une. Para ello se utiliza Ácido Clorhídrico, Ácido Fluorhídrico y Ácido Nítrico, y posteriormente se separa la fracción orgánica de la fracción mineral concentrando la fracción mineral en el fondo de un vidrio de reloj, mientras que la parte orgánica queda suspendida en el agua. La parte orgánica se recupera utilizando filtros de 10 micrómetros de apertura. Las placas de estudio se montan utilizando bálsamo de Canadá.

La distribución estratigráfica de palinomorfos se presentagráficamente en las Tablas I y II. La Tabla I presenta la distribución estratigráfica de palinomorfos de origen continental (i.e., Polen y Esporas), mientras que la Tabla II relaciona los palinomorfos de origen marino (i.e., Dinoflagelados, Microforaminíferos, Escolocodontos, y Acritarcos). En las Planchas fotográficas 1 y 2 se presentan algunos de los palinomorfos más indicativos para la asignación de edades.

Tabla I. Distribución estratigráfica de Polen y Esporas de la Formación Cansona en la quebrada Peñitas.

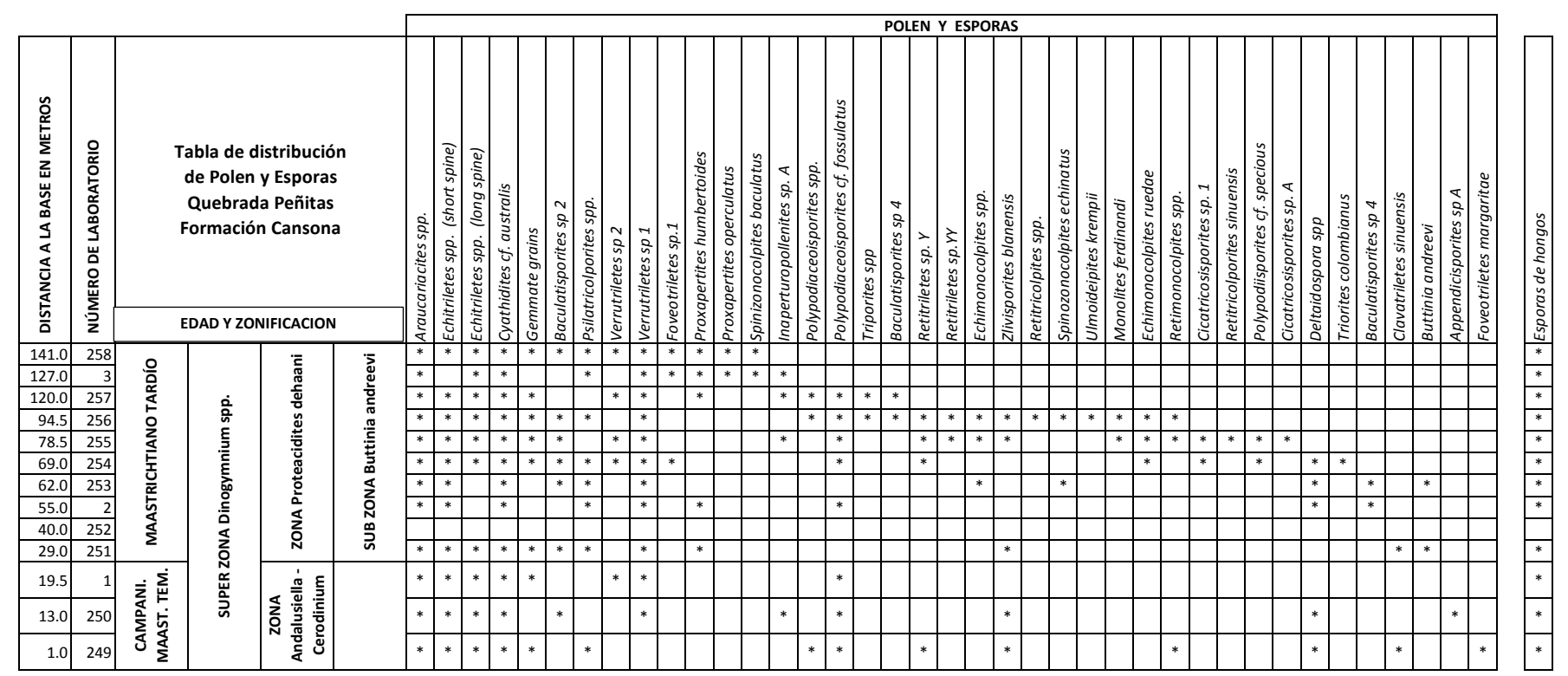

Tabla II. Distribución estratigráfica de Dinoflagelados y otros palinomorfos de la Formación Cansona en la quebrada Peñitas.

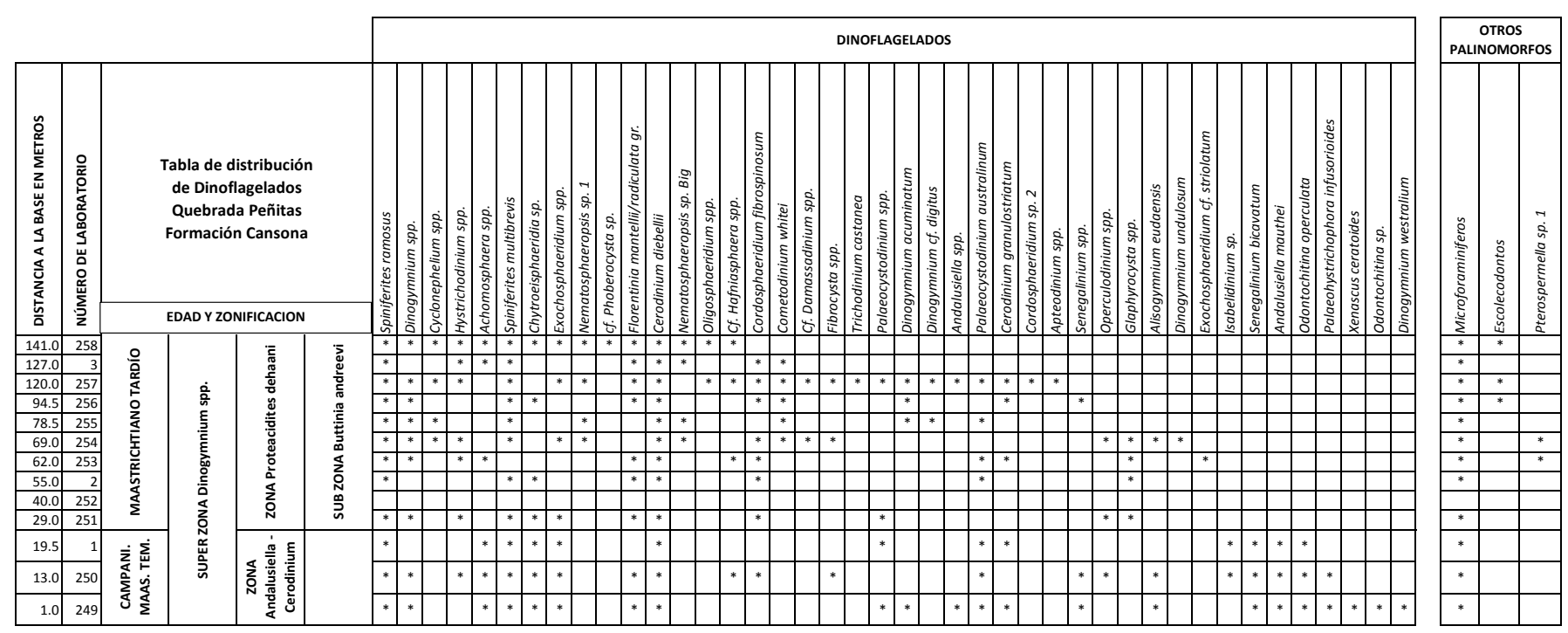




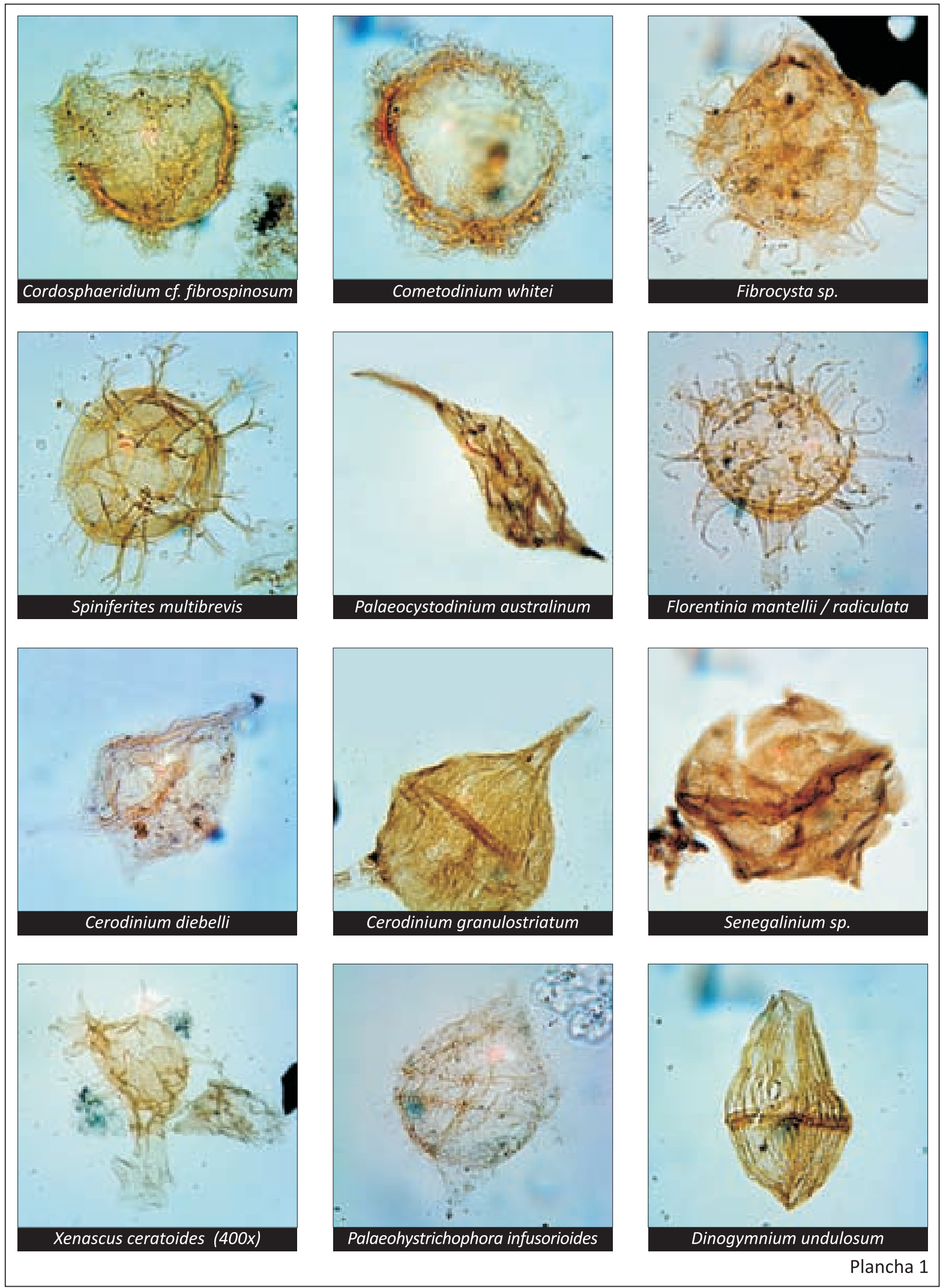



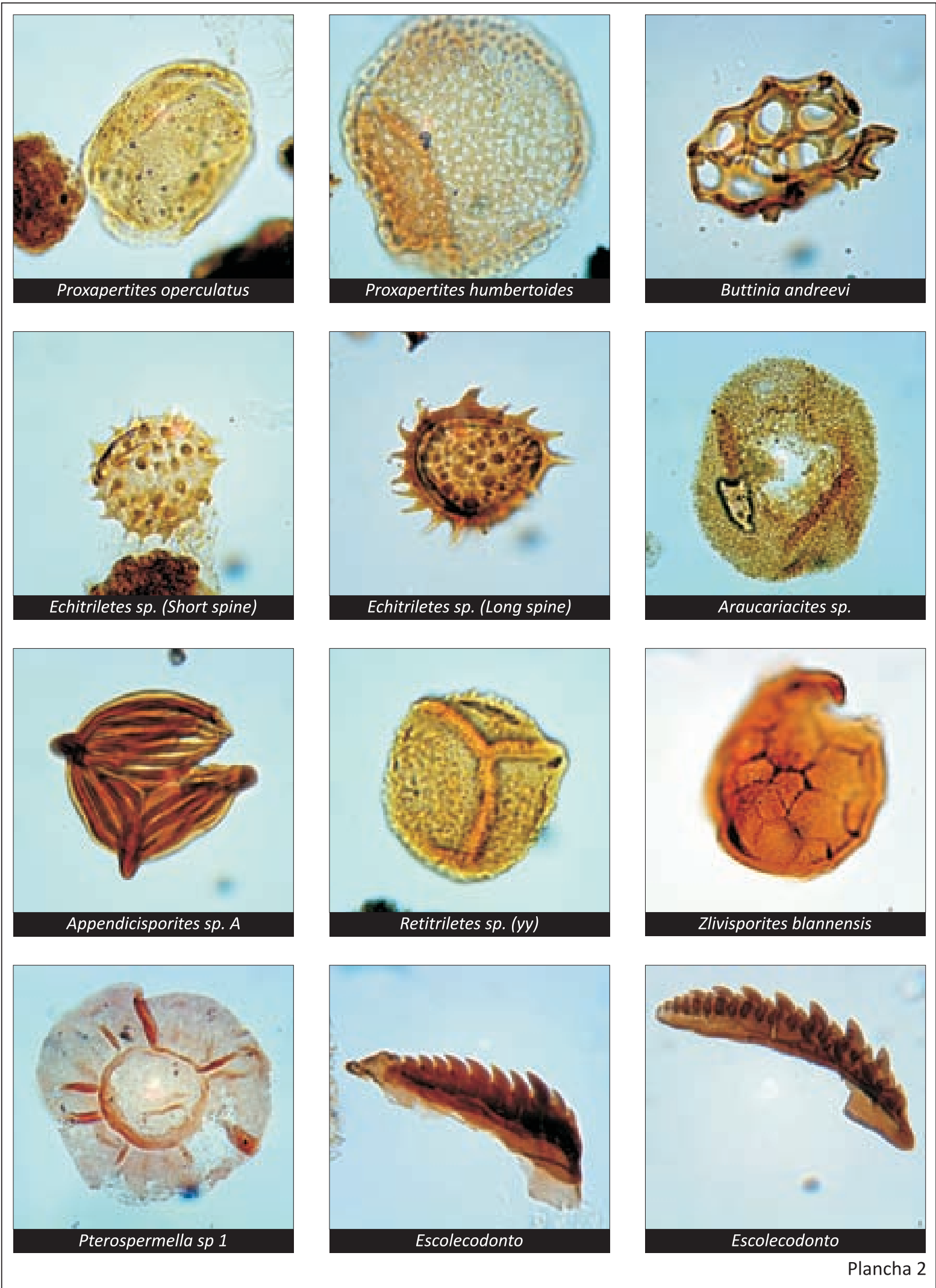
Tabla III. Distribución estratigráfica de microfauna de la Formación Cansona en la quebrada Peñitas.

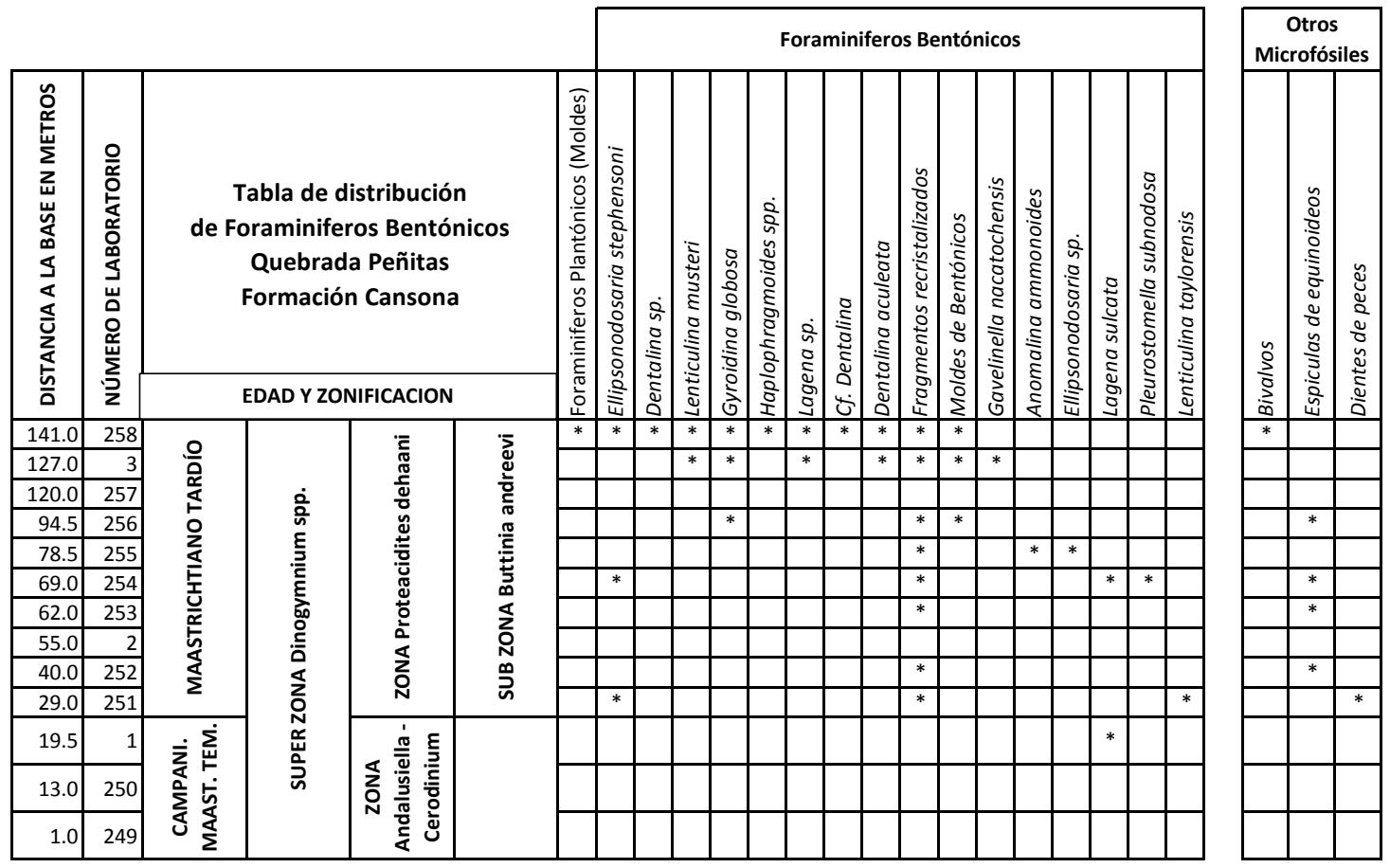

Las muestras para estudios micropaleontológicos se prepararon utilizando métodos convencionales. La Tabla III presenta la distribución estratigráfica de la microfauna encontrada.

\section{Bioestratigrafía}

De todas las muestras preparadas por métodos palinológicos fue posible recuperar muy buenas asociaciones de Esporas Triletes que incluyen esporas de los Géneros Echitriletes, Verrutriletes, Cyathidites, Polypodiaceoisporites y Baculatisporites entre otros. Todas las muestras analizadas correspondientes a esta sección estratigráfica presentan valores altos de esporas de los géneros antes mencionados. La presencia común de estos palinomorfos en todas las muestras analizadas es base para sugerir que estos estratos fueron depositados bajo unas condiciones sedimentarias similares, en las cuales el aporte de elementos de origen continental no solo fue el mismo, sino que fue tambien abundante en todos los casos (Tabla I).

Las asociaciones palinológicas recuperadas incluyen la presencia de granos de polen de las especies Proxapertiteshumbertoides, Spinizonocolpites baculatus, Spinizonocolpites echinatus, Echimonocolpites ruedae y Ulmoideipites krempii entre otros. De gran importancia estratigráfica es la presencia de Buttinia andreevi (Tabla I), la cual presenta su primera aparición en la base del Maastrichtiano Tardío (Sarmiento \& Guerrero, 2000) y su punto de extinción hacia el tope del Maastrichtiano Tardío (Germeraad et al., 1968). La presencia de Buttinia andreevi nos permite por lo tanto determinar una edad Maastrichtiano Tardío para la parte superior (50 a 152 metros) de la sección analizada de la Formación Cansona (Fig.3). Asi mismo, Buttinia andrevii es uno de los elementos más característicos de la zona de Proteacidites dehaanii establecida por Germeraad et al. (1968).

Las muestras analizadas de la Formación Cansona también se caracterizan por presentar abundantes y diversas asociaciones de Dinoflagelados, y pobres asociaciones de Foraminíferos Bentónicos. La presencia de estos elementos de origen marino junto con la abundante presencia de microflora de origen continental nos permite determinar que los sedimentos de la Formación Cansona en la Quebrada Peñitas, se depositaron en un ambiente marino cercano a la línea de costa, el cual recibia un gran aporte de terrígenos.

La parte más inferior de la secuencia estudiada $(0$ - 50 m) se caracteriza por la co-ocurrencia de dinoflagelados de gran importancia estratigráfica tales como:

- Odontochitina operculata y Xenascus ceratoides, palinomorfos cretácicos de amplio rango estratigráfico 
cuyas últimas apariciones se reportan al tope del Maastrichtiano Temprano (Williams \& Bujack, 1985).

- Palaeohystrichophora infusorioides, dinoflagelado cretácico de amplio rango estratigráfico cuya última aparición ha sido reportada en el Maastrichtiano Temprano por Williams \& Bujack (1985), y Williams et al., (1993).

Con base en la presencia de estos dinoflagelados, podemos asignarle a los sedimentos de la parte inferior de la Formación Cansona (0 a 50 metros) una edad "no más joven que Maastrichtiano Temprano”.

Varios de los dinoflagelados que acompañan a O. operculata y a $X$. ceratoides presentan puntos de aparición en la parte superior del Campaniano Tardío, ellos son:

- Spiniferites multibrevis, cuyo punto de aparición se reporta en el límite Campaniano-Maastrichtiano (Schrank, 1987).

- $\quad$ Cerodinium diebelli, presenta su punto de aparición en el Campaniano Tardío (Lentin \& Williams, 1980; Williams \& Bujack,1985).

- Palaeocystodinium australinum, presenta su ocurrencia más baja en la parte más superior del Campaniano (Williams \& Bujack, 1985).

- Cerodinium granulastriatum presenta su ocurrencia más baja en el Campaniano (Sarmiento \& Guerrero, 2000; Schrank, 1987).

- $\quad$ Senegalinium bicavatum y Andalusiella mauthei, los cuales presentan sus puntos de aparición en el Campaniano Tardío (Sarmiento \& Guerrero, 2000).

Teniendo en cuenta lo anteriormente expuesto, podemos concluir que la edad de la parte más baja de la sección analizada de la Formación Cansona (0 a 50 metros) es Campaniano Tardío a Maastrichtiano Temprano (Fig. 3).

En forma general, se observa en las gráficas de distribución de palinomorfos, la disminución de la densidad de los dinoflagelados a medida que se asciende en sentido estratigráfico, así como el incremento de los elementos de origen continental. Esto sugiere una somerización de la cuenca a medida que se asciende estratigráficamente. Esta somerización apoya la idea generalizada de una regresión, que se presenta al final del Cretáceo en la parte Noroccidental de Sur-América.

Asociaciones de palinomorfos similares a las recuperadas de las muestras de la Formación Cansona, en la Quebrada Pe- ñitas, han sido reportadas a lo largo y ancho de la Cordillera Oriental (Fig. 4) por autores tales como:

- $\quad$ Formación Colón: Dueñas (1989); Yepes (2001); Germeraad et al. (1968).

- $\quad$ Formación Molino: Dueñas (1989)

- $\quad$ Formación Mito-Juan: Yepez (2001); Germeraad et al. (1968)

- Grupo Guaduas: Sarmiento (1992); Sarmiento \& Guerrero (2000)

- $\quad$ Formación Umir: Germeraad et al. (1968)

- $\quad$ Formación La Paz: Germeraad et al. (1968)

Lo anteriormente expuesto es base para sugerir la presencia de un ambiente marino somero durante el Cretáceo Tardío, que permitió el depósito de sedimentos similares a lo largo de la Cordillera Oriental, así como en la región del Anticlinal de Chalán, que está localizado al Occidente de la Sutura de Romeral.

\section{Implicaciones paleogeograficas}

El Cinturón de San Jacinto, en donde aflora la Formación Cansona (Anticlinal de Chalán) queda localizado al occidente de la Sutura de Romeral, la cual es considerada el límite entre varios Terrenos Geológicos (Fig. 1). El basamento al occidente de la Sutura de Romeral es de origen volcánico básico, mientras que el basamento al oriente está conformado por rocas ígneas félsicas. La gran mayoría de las reconstrucciones paleogeográficas (Villamil, 1999) consideran que durante el Cretáceo Superior, los Terrenos Geológicos a ambos lados de la Sutura de Romeral evolucionaron geológicamente de forma aislada.

La presencia de afloramientos de sedimentos marinos someros del Cretáceo Tardío en el Anticlinal de Chalán, directamente correlacionables en tiempo y en ambiente de depósito con estratos de formaciones aflorantes al oriente de Romeral, indica claramente que la propuesta de un aislamiento de estos terrenos geológicos durante el Cretáceo Superior no es correcta.

Por primera vez se reporta la presencia de sedimentos pertenecientes a la zona de Proteacidites dehaani (Paleocinturon Tropical) al occidente de la denominada Sutura de Romeral. En la Figura 5, se presenta un esquema geológico regional de la distribución de los sedimentos del Maastrichtiano Tardío en Colombia, en el cual se incluye las áreas de afloramiento de la Formación Cansona. 


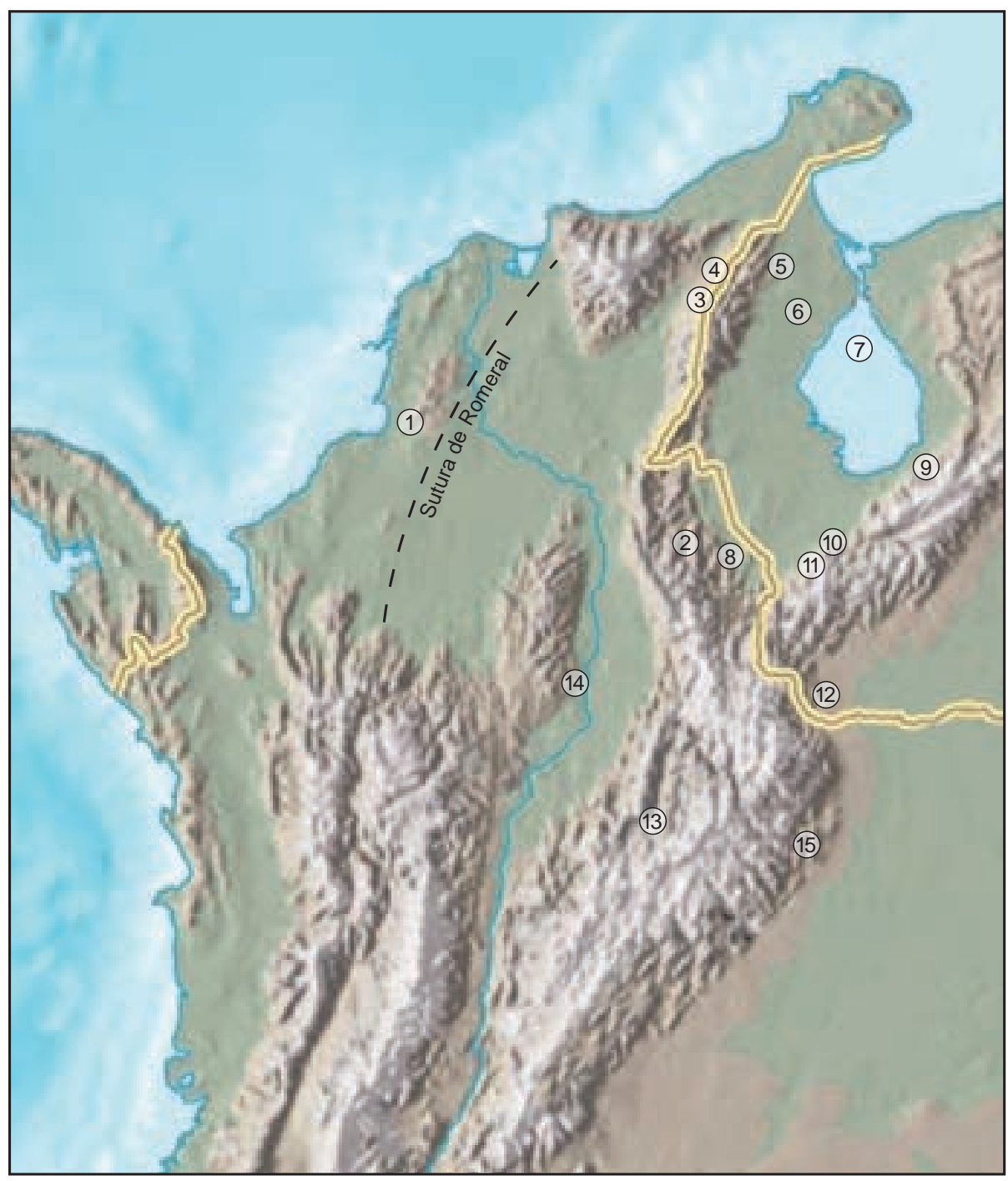

(1) Quebrada Peñitas Fm. Cansona Dueñas \& Gómez

(2) Cuenca Catatumbo Fm. Colón Dueñas 1987

(3) Cuenca Cesar Fm. Molino Dueñas 1987

(4) Sección Río Molino Fm. Colón Yepez (2001)

(5) Sección Riecito Mache Fm. Colón Germeraad etal (1968)
(6) Pozo Concepción 154 La Paz Shale Germeraad etal (1968)

(7) Lago de Maracaibo Área Central Norte La Paz Shale Germeraad etal (1968)

(8) Sección Río Tucuco Fm. Colón Germeraad etal (1968)

(9) Sección Río Mullapas Fm. Colón Germeraad etal (1968)

(10) Sección Río Loro Fm. Colón Mito J uan Yepez (2001)
(11) Quebrada La Mora Fm. Colón Germeraad etal (1968)

(12) Sección Camino Rubio Fm. Colón Mito juan Germeraad etal (1968)

(13) Fm. Guaduas Sarmiento ((1994)

(14) Sección Río Lebrija Fm. Umir Germeraad etal (1968)

(15) Sección Pie de Monte Llanero Fm. Guaduas Sarmiento \& Guerrero (2000)

Figura 4. Distribución de sedimentos pertenecientes a la zona de Proteacidites dehanni Germeraad et al., (1968) 


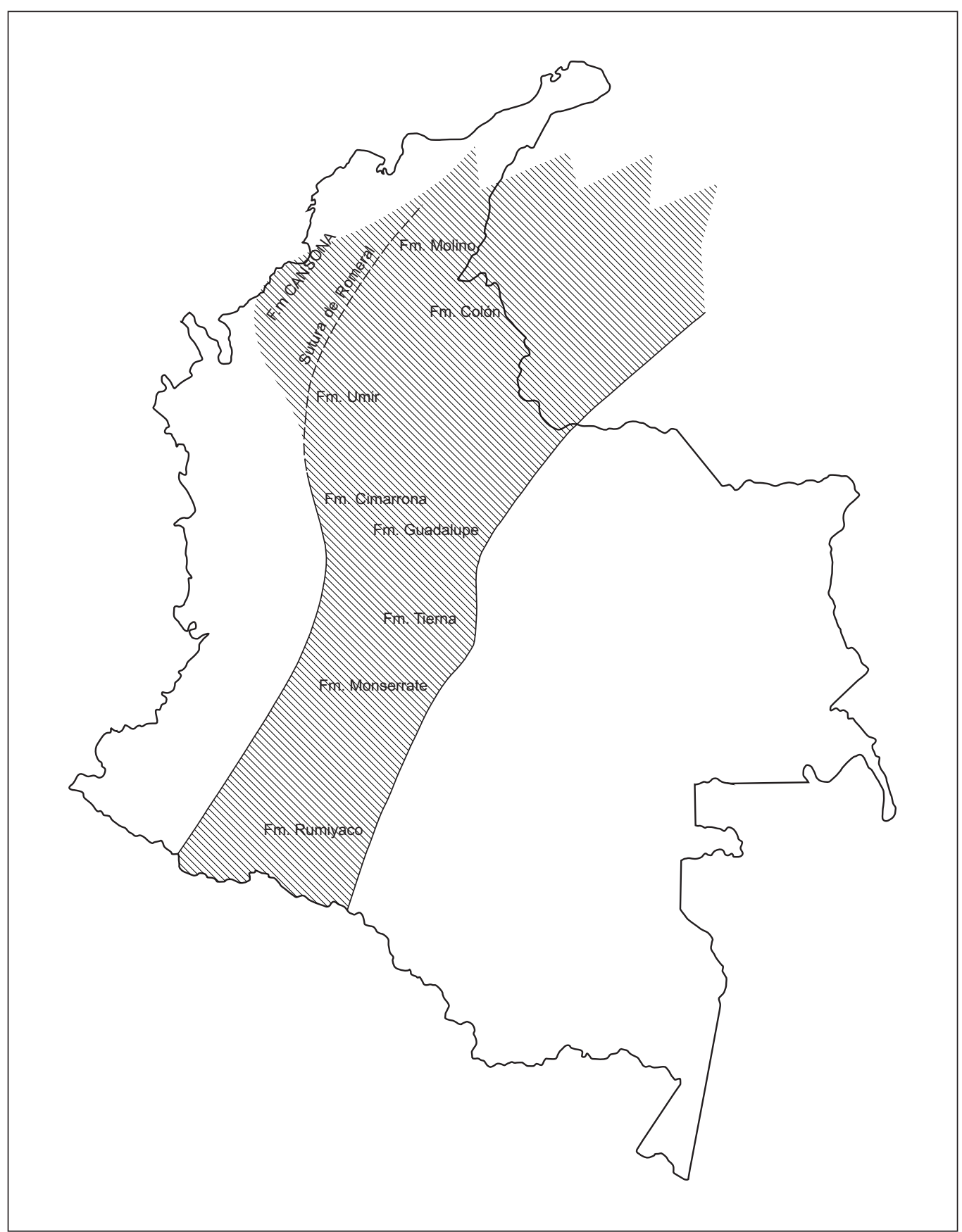

Figura 5. Mapa paleogeográfico del Maastrichtiano. Adaptado de Villamil, 1999 y Higgs, 2009

\section{Conclusiones}

Con base en las asociaciones de palinomorfos recuperadas, se asigna una edad Cretaceo Tardío a los sedimentos de la Formación Cansona, los cuales fueron depositados en un ambiente marino somero cercano a la linea de costa. Estas asociaciones de palinomorfos son muy similares a las repor- tadas en sedimentos correlacionables en edad y ambientes de deposito aflorantes a lo largo y ancho de la Cordillera Oriental.

Desde el punto de vista paleogeográfico, la presencia de los sedimentos de la Formación Cansona al occidente de la Sutura de Romeral indica que los Terrenos Geologicos a am- 
bos lados de la Sutura de Romeral no evolucionaron durante el Cretaceo Tardío en forma independiente. Por lo tanto, es muy importante entender qué fuerzas tectonicas aislaron el bloque del Anticlinal de Chalán, y como actuaron dichas fuerzas durante la evolución del Caribe colombiano.

\section{Agradecimientos}

Los autores desean agradecer a la Agencia Nacional de Hidrocarburos de Colombia (A.N.H.) y a BIOSS SAS el permitirnos publicar este trabajo. Al mismo tiempo agradecemos los oportunos y acertados comentarios que hemos recibido del comité editorial de la revista ACCEFYN.

\section{Bibliografía}

Bioss, Ltda. (2005) Interpretación geológica de la cuenca del Valle Inferior del Magdalena. Reportes internos. Bogotá.

Clavijo, J.: Barrera, R. (2001) Geología de las Planchas 44 Sincelejo y 52 Sahagún. Escala 1:100.000 Memoria Explicativa. INGEOMINAS.

Dueñas, H. (1989) Asociaciones palinológicas de las Formaciones Colon y Molino Nor-Oriente Colombiano. Bol. IG-USP, Publicación Especial 7:173-181.

Dueñas, H.; Duque Caro, H. (1981) Geología del Cuadrángulo F - 8 Planeta Rica. Boletin Geológico INGEOMINAS 24(1):1 - 35.

Duque Caro, H. (1972) Ciclos tectónicos y sedimentarios en el Norte de Colombia y sus relaciones con la Paleoecología. Boletín Geológico INGEOMINAS 19(3):1 - 23.

Duque Caro, H.; Guzman, G.; Hernandez, R. (1996) Mapa Geológico de la Plancha 38, Carmen de Bolívar. INGEOMINAS, Bogotá.

Germeraad, J.H.; Hopping, C.A.; Muller, J. (1968) Palynology of Tertiary sediments from Tropical areas. Review of Palaeobotany and Palynology, 6: 189 - 348 .

Guerrero, J.; Sarmiento, G. (1996) Estratigrafía física, Palinológica, Sedimentológica y Secuencial del Cretacico Superior y Paleoceno del
Piedemonte Llanero. Implicaciones en Exploración Petrolera. Geología Colombiana 20:3 - 66. Departamento de Geociencias, Universidad Nacional de Colombia.

Guzman, G. (2007) Stratigraphy and Sedimentary Environment and Implications in the Plato Basin and the San Jacinto Belt Northwestern Colombia. Doctoral Thesis, University of Liege.

Lentin, J.K.; Williams, G.L. (1980) Dinoflagellate provincialism with emphasis on Campanian Peridiniaceans. American Association of Stratigraphic Palynologists Foundation (No. 7),p. 1 - 47.

Sarmiento, G. (1992) Palinología de la Formación Guaduas - estratigrafía y sistemática. Boletín Geológico INGEOMINAS 32:45 - 126.

Sarmiento, G.; Guerrero, J. (2000) Palinología del Santoniano Tardío al Maastrichtiano del Piedemonte Llanero Colombiano. Correlación con el Paleocinturón Tropical. Geología Colombiana 25:111 - 147.

Schrank, E. (1987) Paleozoic and Mesozoic Palynomrphs from northeast Africa (Egypt and Sudan) with special reference to Late Cretaceous pollen and dinoflagellates. Berliner Geowissenschaftliche Abhandlungen (A), 75:249 - 310 .

Villamil, T. (1999) Campanian - Miocene tectonostratigraphy, depocenter evolution and basin development of Colombia and Western Venezuela.Palaeogeography, Palaeoclimatology, Palaeoecology, 153: 239 -275 .

Williams, G.L.; Bujack, J.P. (1985) Mesozoic and Cenozoic Dinoflagellates. In Bolli, H. M., Saunders, J.B. and Perch-Nielsen, K. (eds). Plankton Stratigraphy. Cambridge University Press, Cambridge, p. $847-964$.

Williams, G.L.; Stover, L.E.; Kidson, E.J. (1993) Morphology and Stratigraphic ranges of selected Mesozoic - Cenozoic dinoflagellate taxa in the Northern Hemisphere.Geological Survey of Canada. Paper 92(10): 1 - 37 .

Yepes, O. (2001) Maastrichtian - Danian Dinoflagellates cyst Bioestratigraphy and Biogeography from two Equatorial sections I Colombia and Venezuela. Palynology, 25:217 - 249.

Recibido: febrero 20 de 2013

Aceptado: 10 de marzo de 2014 